\title{
STABILITY OF THIN-WALLED SQUARE TUBES WITH INTERMEDIATE STIFFENERS UNDER TORSION
}

\author{
ZBigniew KoŁakowski, JaCek Jankowski \\ Lodz University of Technology, Department of Strength of Materials, Lodz, Poland \\ e-mail: zbigniew.kolakowski@p.lodz.pl; jacek.jankowski@p.lodz.pl
}

\begin{abstract}
The present paper deals with the buckling of thin-walled square tubes with intermediate stiffeners under torsion when the shear lag phenomenon and distortional deformations are taken into account. A plate model (2D) was adopted for the tube. The tubes were assumed to be simply supported at their ends. The values of critical loads of the tubes were determined with three methods, namely: analytical-numerical method (ANM), finite difference method (FDM) and finite element method (FEM).

Keywords: torsion, thin-walled tubes, intermediate stiffeners, analytical-numerical method (ANM), finite difference method (FDM), finite element method (FEM)
\end{abstract}

\section{Introduction}

Thin-walled cold-formed steel (CFS) members (columns, beams and girders) are very widely used in constructions. The capacity to resist loads in thin-walled beams is limited not only by their strength but, first of all, by stability.

In the stability analysis of thin-walled structures, the following numerical methods are often applied: finite strip method (FSM), finite element method (FEM) and global beam theory (GBT).

The FSM is broadly used for analysis of elastic stability due to very high computational capabilities, low computational costs and easy implementation to thin-walled elements. The method is restricted mainly to simple geometries and boundary conditions. Therefore, a series of new variants or enhancements are needed to be introduced to the FSM.

In the FEM, two sorts of analyses are usually carried out: (i) elastic stability analysis, that is to say, the eigenproblem, and (ii) nonlinear post-buckling analysis to determine the performance curve in the whole range of structure operation. Papers (Adany, 2018; Adany et al., 2018) deal with the constrained finite element method (cFEM) employed for the buckling analysis of columns with opened cross-sections.

An application of the GBT allows for determination of participation of various buckling modes within the whole range of structure operation. Understanding of the complex phenomenon of coupled buckling is thus possible. In (Martins et al., 2017), distortional failure of steel beams simply supported was presented with the direct strength method (DSM). In (Martins et al., 2018), the interactive buckling of a lipped channel was analysed with the generalized beam theory (GBT). The development in the theory of coupled instabilities of thin-walled structures was discussed in (Hancock, 2018).

In (Rendal et al., 2017), a semi-analytical finite strip method (SAFSM) for the buckling analysis of thin-walled structures under general loading, including shear, was presented. A local and distortional buckling analysis of thin-walled tubes with a regular convex polygonal section under uniform torsion with the cFSM was studied in (Rendal et al., 2018). Attention was also paid to the necessity to account for all non-linear components of the in-plane shear strain, which 
resulted in membrane instabilities. Stability of thin-walled tubes with regular cross-sections, from triangle to octagon, and the same circumference, subjected to torsion, was analysed. The full Green's strain tensor within nonlinear stability problems was already considered in (Kolakowski and Krolak, 2006; Kolakowski and Mania, 2013; Kolakowski and Teter, 2000; Królak et al., 2001; Teter and Kołakowski, 2003, 2004).

In (Królak et al., 2001), stability and load carrying capacity of thin-walled orthotropic tubes under torsion and compression or bending was analysed. Regular polygons, from square to icosagon, were considered. The problem was solved with the analytical-numerical method (ANM) and the finite element method (FEM). In the ANM described in (Królak et al., 2001), the full Green's strain tensor for thin-walled plates, the second Piola-Kirchhoff's stress tensor, the shear lag phenomenon, an effect of cross-sectional distortions, as well as coupled conditions between all the walls of structures were included.

The present paper was inspired by Rendal et al. (2018). Stability of steel square and octagonal tubes of the same circumference but different lengths, subject to torsion, was analysed with the ANM discussed in (Królak et al., 2001). In the case of square tubes, cross-sections with C- and L-shaped intermediate stiffeners and without stiffeners were considered. Intermediate stiffeners divide each plate into two smaller plate bands and, depending on the stiffener shape and dimensions, reinforce the whole plate (Kolakowski and Teter, 2000; Teter and Kołakowski, $2003,2004)$. When the plate is bent at least by 45 degrees, this increases significantly the plate stiffness. The results obtained for "smooth" cross-sections were compared to the FEM results attained with the ANSYS software package and with the results from the finite difference method (FDM).

\section{Formulation of the problem}

In (Królak et al., 2001), the analytical-numerical method was described in detail. In the investigations discussed here, steel tubes were subject solely to torsion. Thus, only the following crucial assumptions are presented below.

Prismatic thin-walled tubes built of plates connected along longitudinal edges were considered. The tubes were simply supported at their ends (Królak et al., 2001). In order to account for all buckling modes, a plate model (i.e., 2D) of thin-walled structures was applied. Moreover, it was assumed that the material the structure obeyed Hooke's law.

For each plate component, precise geometrical relationships (i.e., full Green's strain tensor) were assumed in order to consider both out-of-plane and in-plane bending of the $i$-th plate (Kolakowski and Krolak, 2006; Kolakowski and Mania, 2013; Kolakowski and Teter, 2000; Królak et al., 2001; Teter and Kołakowski, 2003, 2004)

$$
\begin{aligned}
& \varepsilon_{x i}=u_{i, x}+\frac{1}{2}\left(w_{i, x}^{2}+v_{i, x}^{2}+u_{i, x}^{2}\right) \quad \varepsilon_{y i}=v_{i, y}+\frac{1}{2}\left(w_{i, y}^{2}+u_{i, y}^{2}+v_{i, y}^{2}\right) \\
& \varepsilon_{x y i}=\gamma_{x y i}=u_{i, y}+v_{i, x}+w_{i, x} w_{i, y}+u_{i, x} u_{i, y}+v_{i, x} v_{i, y}
\end{aligned}
$$

and

$$
\kappa_{x i}=-w_{i, x x} \quad \kappa_{y i}=-w_{i, y y} \quad \kappa_{x y i}=-2 w_{i, x y}
$$

where: $u_{i}, v_{i}, w_{i}$ - components of the displacement vector of the $i$-th plate along the $x_{i}, y_{i}, z_{i}$ direction, respectively, and the plane $x_{i} y_{i}$ overlaps the central plane before its buckling.

The solution to the problem was assumed in the form of trigonometric series along the longitudinal direction of the tubes, whereas the exact transition matrix method and the numerical method of the transition matrix using Godunov's orthogonalization were used along the transverse direction. 
The computations within the FDM and the FEM were conducted in the present study as well. In the FDM, according to the classic theory of thin isotropic plates, it was assumed that the two last terms were neglected in each expression describing strains (2.1). The FDM consists in substitution of respective derivatives of displacements in the equilibrium equations, plate interaction conditions and boundary conditions by the corresponding differential quotients. In the equilibrium equations, central differential quotients were assumed, whereas in the remaining equations of the problem - left-hand-side and right-hand-side differences. When derivatives were substituted by quotients, a linear system of algebraic equations was formulated. This system corresponded to the problem of eigenvalues and allowed for determination of values of critical loads and their corresponding buckling modes. In the calculations of, e.g., the square cross-section, the number of assumed nodes was equal to 1044.
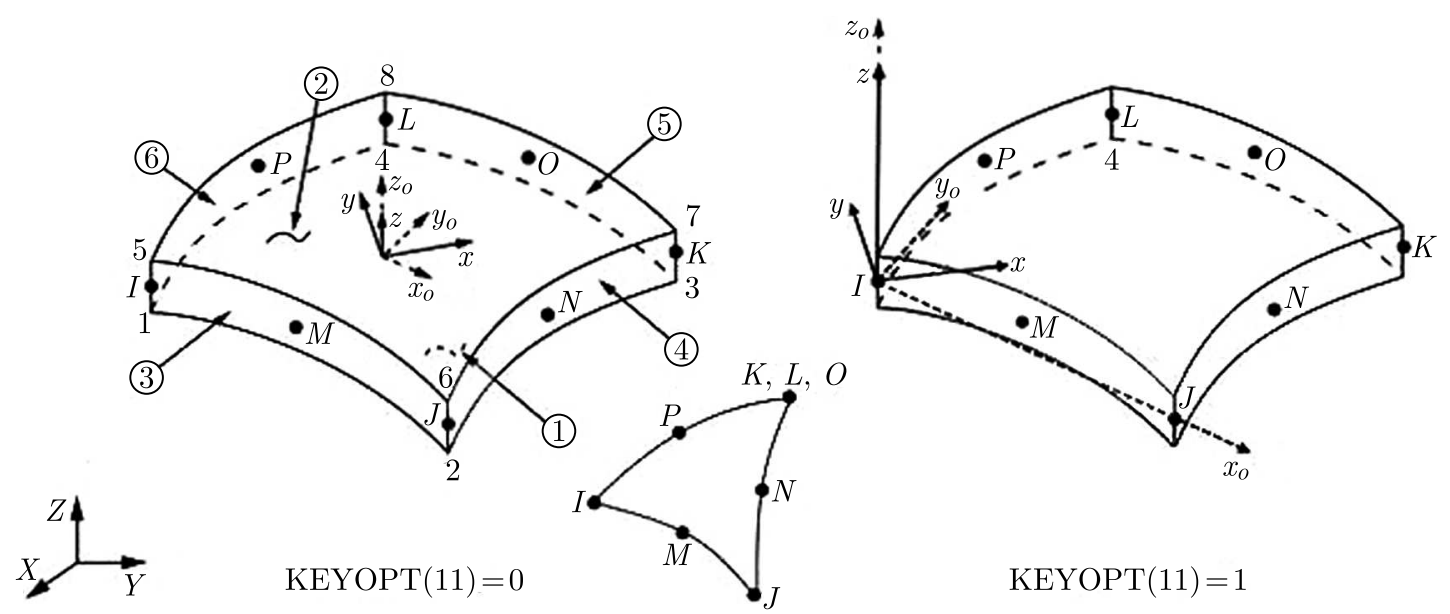

Fig. 1. SHELL281 element

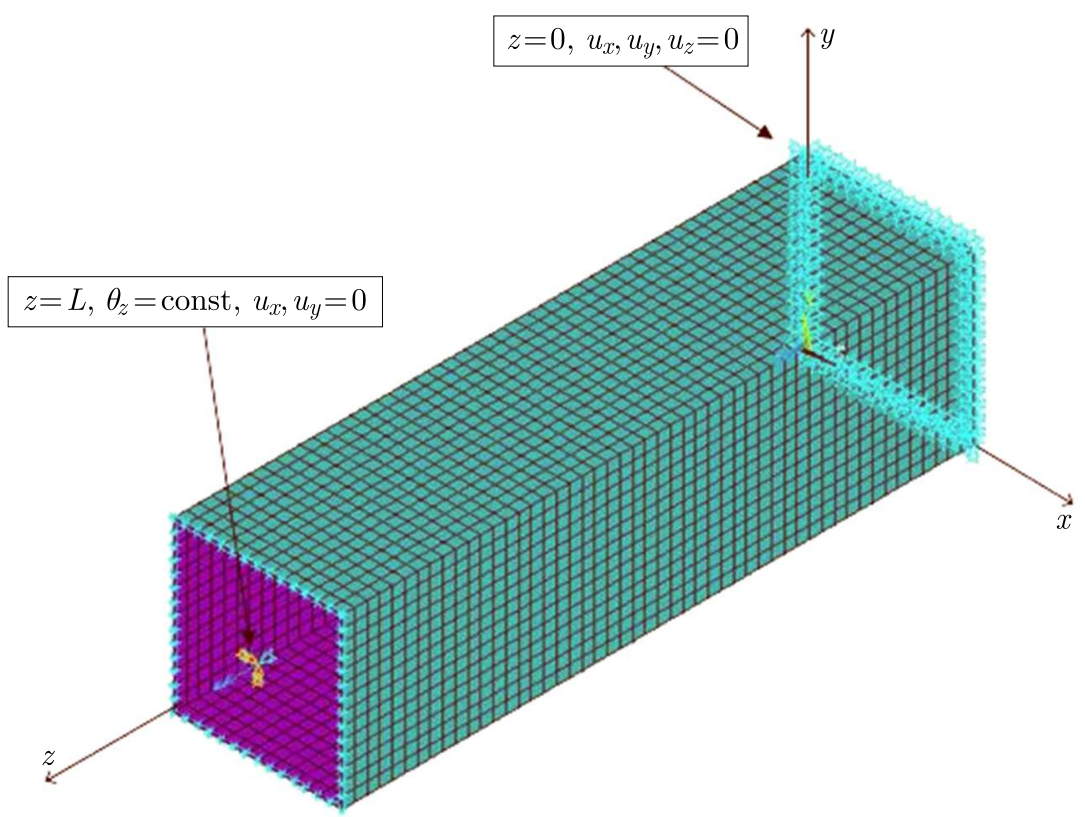

Fig. 2. FEM numerical model of the square tube

In order to validate the calculation results attained with the finite difference method (FDM), an ANSYS package based on the FEM was used. The SHELL281 (second order) finite element was applied in modelling. Figure 1 shows this shell element. An example of the numerical model 
of the square profile subject to constrained torsion together with boundary conditions is depicted in Fig. 2. One profile end was fixed, whereas the second one was subject to loading in the form of the moment applied to the node lying in the centre of gravity of the cross-section. The load was transferred on the profile nodes (in the torsion plane) by MPC (contact) elements. For instance, the numerical model of the profile, whose length is equal to $L=500 \mathrm{~mm}$, includes 2857 nodes.

\section{Analysis of the calculation results}

Square tubes with C-shaped (Fig. 3a) and V-shaped (Fig. 3b) intermediate stiffeners of the same dimensions $b_{s}$ or without stiffeners but with an octagonal cross-section (Fig. 3c), subject to torsion, were investigated. The following geometrical dimensions of the cross-sections under consideration were assumed (Fig. 3):

- square cross-section without stiffeners: $b=100 \mathrm{~mm}, b_{s}=0 \mathrm{~mm}, t=2 \mathrm{~mm}$;

- square cross-section with stiffeners: $b=100 \mathrm{~mm}, b_{s}=8 \mathrm{~mm}, t=2 \mathrm{~mm}$;

- regular octagonal cross-section: $b=50 \mathrm{~mm}, t=2 \mathrm{~mm}$;

and the following material constants: $E=200 \mathrm{GPa}, \nu=0.3$.

(a)

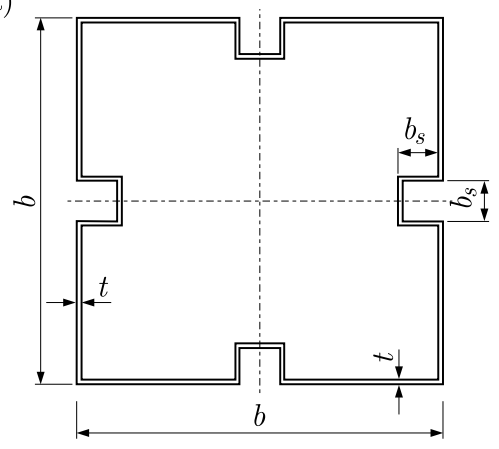

(b)

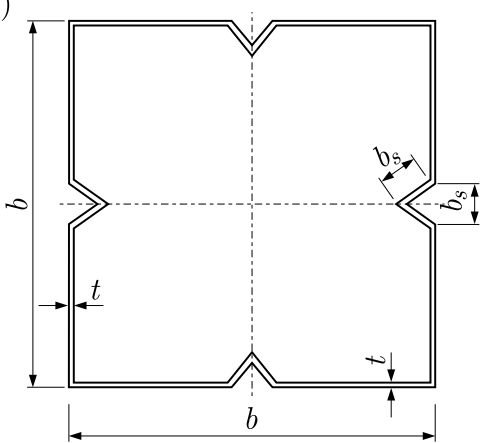

(c)

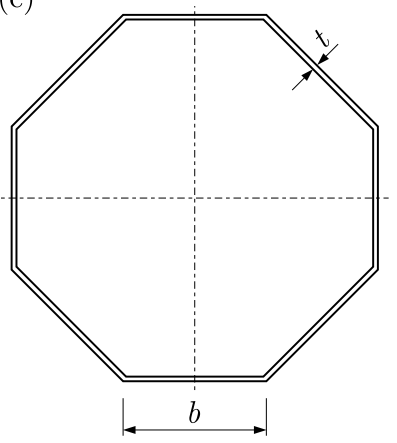

Fig. 3. Cross-sections of thin-walled tubes: (a) square with C-stiffeners, (b) square with V-stiffeners,

(c) regular octagonal

The computations were performed with the ANM code developed in (Królak et al., 2001). The results were compared to the FDM and the FEM for "smooth" tubes (i.e., for square and octagonal profiles) of a few selected lengths and the FEM for square tubes with C-stiffeners. The values of tangential critical stresses for various lengths $L$ of the tubes are listed in Table 1 . The values of critical moments can be determined on the basis of commonly known formulas for the material strength analysis.

In (Królak et al., 2001), the ANM solution was predicted in the form of trigonometric series. The developed code allows a large number of terms to be considered in the series. For the ANM results shown in Table 1, the following numbers of terms in the series $m$ were assumed for various lengths: for $L=50 \mathrm{~mm}-7, L=100 \mathrm{~mm}-11, L=200 \mathrm{~mm}-11, L=500 \mathrm{~mm}-21$, $L=1000 \mathrm{~mm}-21, L=2000 \mathrm{~mm}-21$. The analysis of the convergence of the results of critical loads for $m \pm 3$ has been carried out and a very good agreement of the values has been attained.

The most unexpected results are obtained for the square cross-section of tubes for the ANM. For these reasons, the results were given in the Appendix for subsequent set values in the series $m$. As can be seen from the Appendix, the results of buckling stresses $\tau_{c r}$ stabilize with the increase of $m$ and no signs of numerical errors have been noticed.

If the results from the ANM are compared, it can be seen that the lowest critical values are obtained for the "smooth" square cross-section, then for V-stiffeners, whereas the highest for C-stiffeners. In the case of the octagon, the values of critical loads are between the values for 
Table 1. Critical loads $\tau_{c r}$ of steel tubes of various lengths

\begin{tabular}{|c|c|c|c|c|c|c|c|}
\hline \multirow{3}{*}{ Cross-sections of tubes } & \multirow{2}{*}{ Methods } & \multicolumn{6}{|c|}{ Buckling stresses $\tau_{c r}[\mathrm{MPa}]$} \\
\cline { 3 - 8 } & & 50 & 100 & 200 & 500 & 1000 & 2000 \\
\cline { 3 - 8 } & ANM & 1637 & 479 & 198 & 140 & 131 & 129 \\
\hline \hline \multirow{5}{*}{ Square } & FDM & - & - & 495 & 417 & 431 & 529 \\
\cline { 2 - 8 } & FEM & - & - & 558 & 428 & 410 & 408 \\
\cline { 2 - 8 } & {$[11]$} & 675 & 375 & 375 & 375 & 375 & 375 \\
\hline \multirow{3}{*}{ Regular octagon } & ANM & 2871 & 1637 & 990 & 703 & 510 & 353 \\
\cline { 2 - 8 } & FDM & - & - & 1310 & 895 & 730 & - \\
\cline { 2 - 8 } & FEM & - & - & 1174 & 785 & 547 & - \\
\cline { 2 - 8 } & {$[11]$} & 1500 & 1075 & 650 & 400 & 335 & 335 \\
\hline \multirow{2}{*}{ Square - C-stiffeners } & ANM & 3474 & 1786 & 1177 & 794 & 486 & 442 \\
\cline { 2 - 8 } & FEM & 3207 & 2004 & 1189 & 832 & 592 & 473 \\
\hline Square - V-stiffeners & ANM & 2917 & 1133 & 758 & 539 & 464 & 417 \\
\hline
\end{tabular}

[11] - Rendal et al. (2018)

V-stiffeners and C-stiffeners, except for three cases, namely: for $L=50 \mathrm{~mm}$ and the two longest tubes.

Higher values of $\tau_{c r}$ for C-shaped intermediate stiffeners can be explained by higher flexural rigidity than in the case of V-stiffeners. Comparing the results for the $\mathrm{C}$ - and V-shaped stiffeners and the octagon, one can state that the C-stiffener yields higher values of $\tau_{c r}$ than the octagon, that is to say, this shape of the stiffener is more rigid than the bending of the adjoining walls in the octagon, except $L=1000 \mathrm{~mm}$. The lowest values of $\tau_{c r}$ were attained for V-stiffeners, except the case of $L=2000 \mathrm{~mm}$. The stiffener shape plays a less and less important role with an increase in the tube length $L$ for $L \geqslant 1000 \mathrm{~mm}$.

For C-stiffeners, the results of calculations conducted with the FEM have been presented as well. A very close agreement between the ANM and FEM results has been obtained. The maximum difference equals 1.2 .

In Table 1, the ANM results have been compared to the FDM and FEM results for "smooth" tubes for a few selected lengths. The critical load $\tau_{c r}$ for the FEM decreases both for the square and octagonal cross-section with an increase in length $L$. A similar situation takes place with the values of $\tau_{c r}$ from the FDM for the octagon. For the square cross-section, the lowest value of $\tau_{c r}$ was attained for $L=500 \mathrm{~mm}$, and the highest one for $L=2000 \mathrm{~mm}$.

For the square cross-section, the values of $\tau_{c r}$ for the ANM are 2.5-4 times lower than the values obtained from the FDM and the FEM, whereas for the case of the octagonal cross-section, the differences from various methods are lower as they do not exceed 1.5 times. The outcomes from the FEM are lower than from the FDM. It can be caused by the fact that the strains were assumed according to the classic theory of thin-walled beams in the FDM, which was confirmed by the conclusions in (Rendal et al., 2018). The SHELL281 (second order) finite element not covering all these components of the full strain tensor has been applied in the FEM.

In (Rendal et al., 2018), the results are depicted on diagrams. For the sake of this paper, the lowest approximate values of the critical loads $\tau_{c r}$ for the "smooth" square (Fig. 4 in (Królak et al., 2001)) and for the octagon (Fig. 12 in (Rendal et al., 2018)) were read. The values of $\tau_{c r}$ are listed in Table 1 as well. For the square cross-section, the values $\tau_{c r}$ attained in (Rendal et al., 2018) are constant in practice for the lengths under consideration, except $L=50 \mathrm{~mm}$. For $L=50 \mathrm{~mm}$, the critical load is 2.4 times lower than for the ANM, whereas for $L=100 \mathrm{~mm}-$ 1.3 times lower. For the remaining lengths, lower values of $\tau_{c r}$ were obtained with the ANM. In the case of the octagon, values of $\tau_{c r}$ (Rendal et al., 2018) decreased with an increase in the 
tube length. They are up to 2 times lower than the ANM for the length $L \leqslant 1000 \mathrm{~mm}$, whereas for $L=2000 \mathrm{~mm}$, they are only 1.1 times lower.

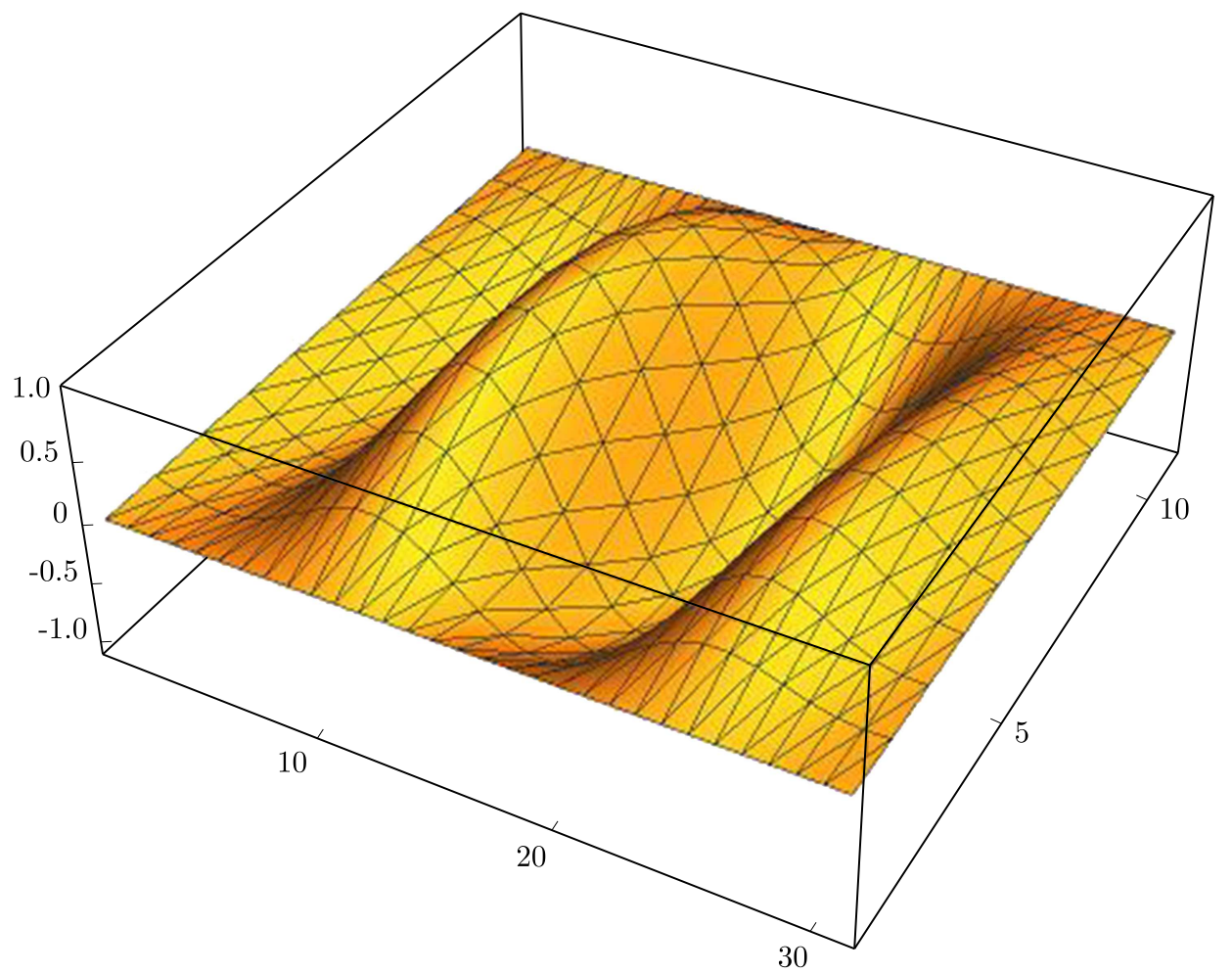

Fig. 4. Buckling mode of a single wall of the square profile of the length $L=500 \mathrm{~mm}$ (FDM)

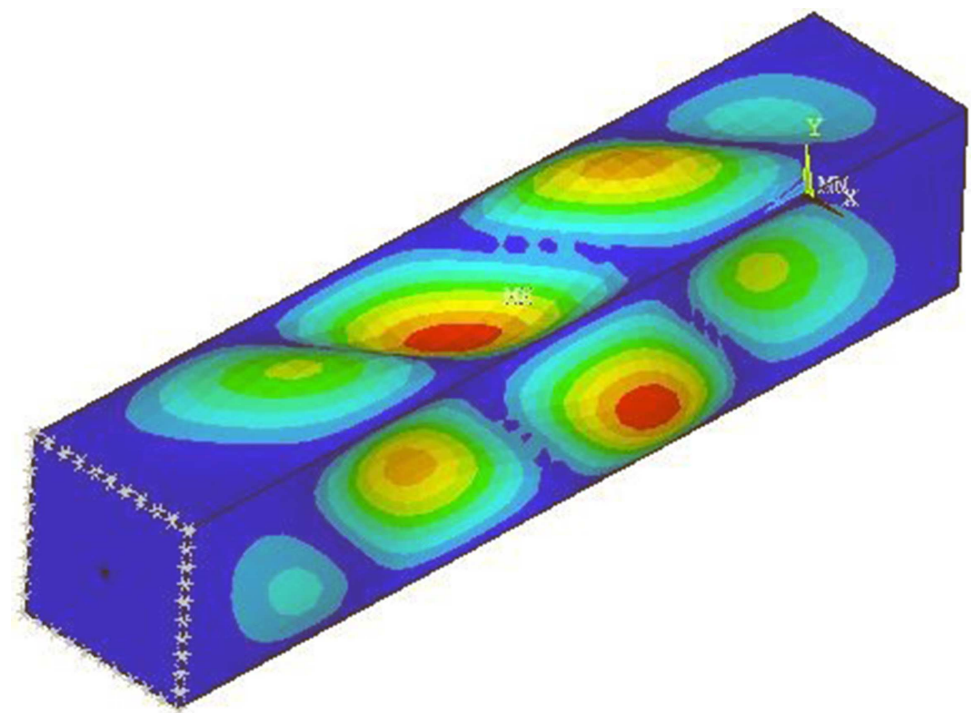

Fig. 5. Buckling mode of the square profile of the length $L=500 \mathrm{~mm}$ (FEM)

In Figs. 4 and 5, the buckling modes for "smooth" square tubes determined with the FDM and the FEM for $L=500 \mathrm{~mm}$ are presented. In Figs. 6 and 7, analogous plots for $L=1000 \mathrm{~mm}$ are depicted. In the case of the FDM, the buckling modes for a single wall are shown. As can be easily seen, the numbers of halfwaves along the longitudinal direction obtained with both the methods are the same for the given length. 


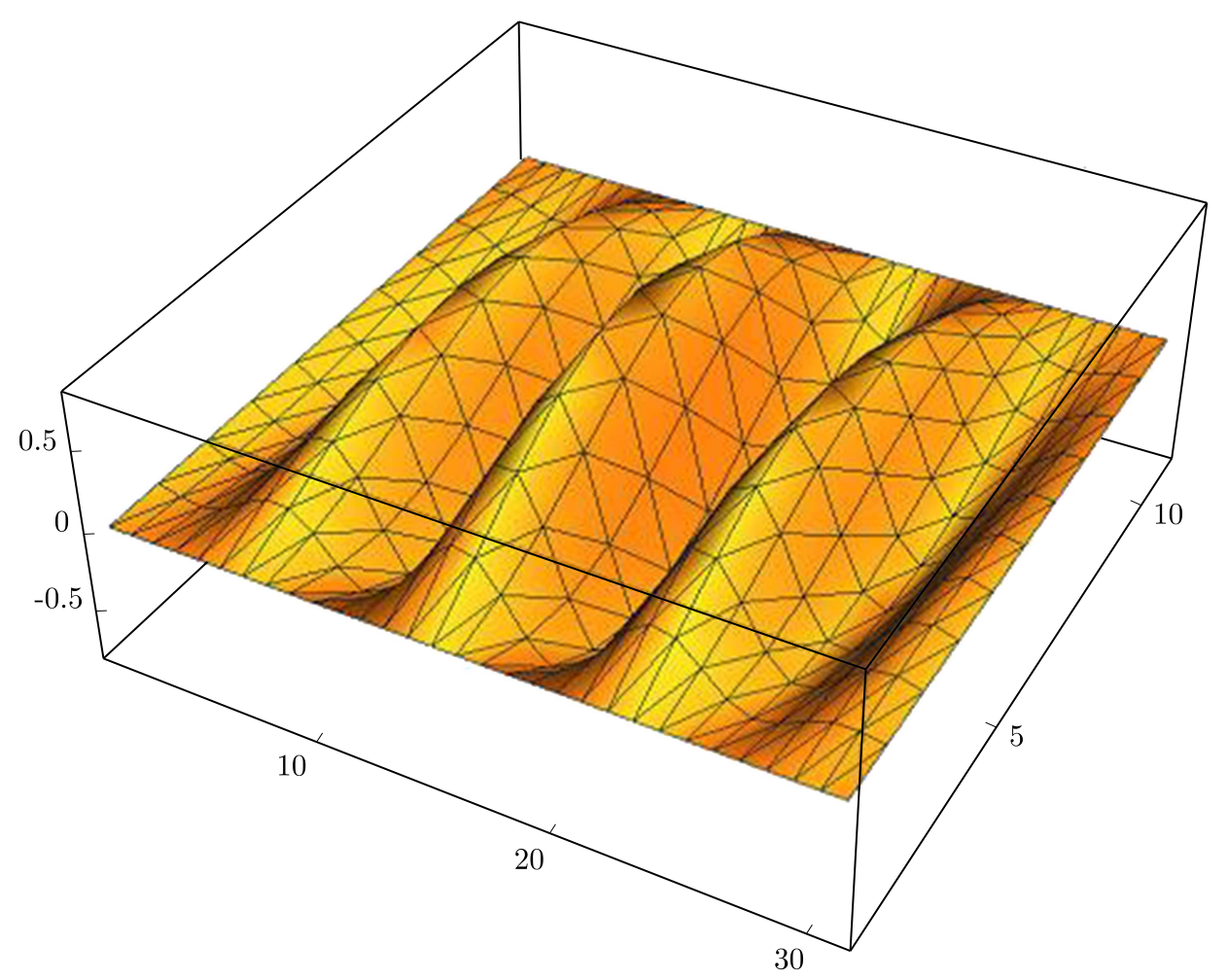

Fig. 6. Buckling mode of a single wall of the square profile of the length $L=1000 \mathrm{~mm}$ (FDM)

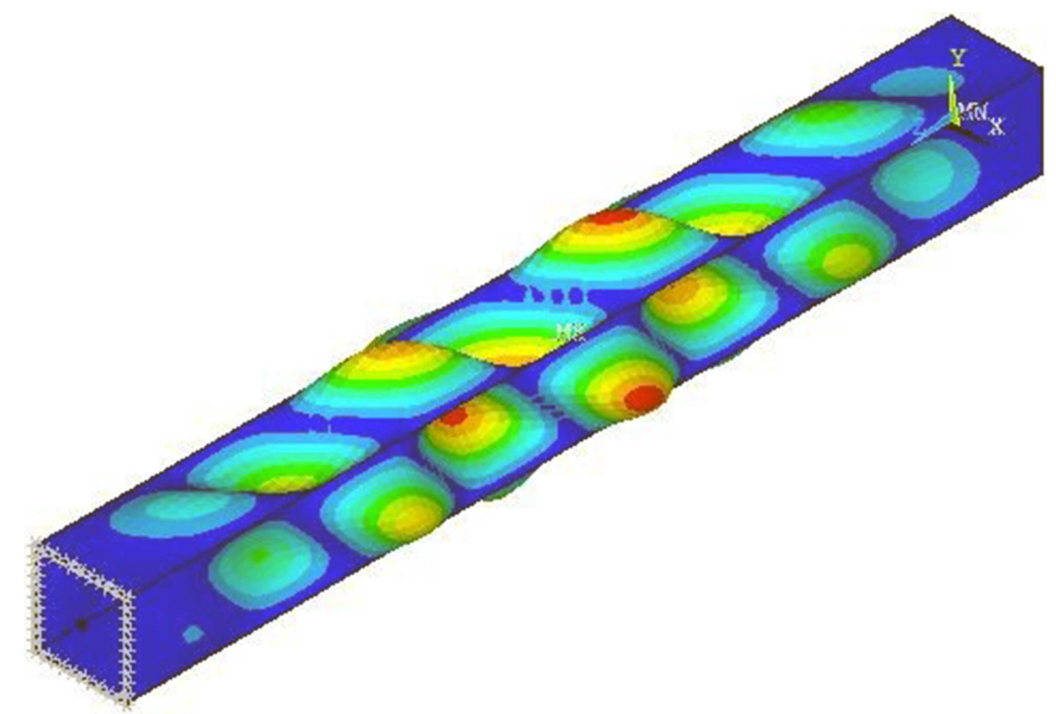

Fig. 7. Buckling mode of the square profile of the length $L=1000 \mathrm{~mm}$ (FEM)

The above considerations draw special attention to different behaviour of the "smooth" square tube when compared with the octagonal tube for all the results under analysis. The FDM and FEM results for various lengths $L$ are of similar nature.

An introduction of intermediate stiffeners for the square cross-section results in a considerably higher critical loads, which are close to the values attained for the octagonal tube.

The presented results indicate that an analysis of causes of such significant discrepancies is needed. The coefficients at each nonlinear term (2.1) assumed in the ANM can easily facilitate this task by zeroing various combinations of these terms and tracking the effects of alternations on the values of $\tau_{c r}$. 


\section{Conclusions}

Constrained torsion of thin-walled square tubes with C- and V-shaped intermediate stiffeners and without them but with an octagonal cross-section was analysed. In the ANM, the shear lag phenomenon and distortional deformations were taken into account. Intermediate stiffeners increased significantly the values of critical loads, which were close to the results for the octagonal tube of the same circumference. The presented results of computations attained with three various methods, namely: ANM, FDM and FEM, were compared to the results listed in (Rendal et al., 2018). Considerable differences in the obtained outcomes were pointed out. The present study is to be treated as an initial analysis of the problem under consideration. Therefore, it requires further comprehensive analysis.

\section{Appendix}

Table A1 shows the critical load values $\tau_{c r}$ obtained for the square cross-section of tubes in the case of the ANM for subsequent numbers of terms in the series $m$.

Table A1. Critical loads $\tau_{c r}$ of steel square tubes using ANM

\begin{tabular}{|c|c|c|c|c|c|c|}
\hline \multirow{2}{*}{$L[\mathrm{~mm}]$} & \multicolumn{6}{|c|}{ Buckling stresses $\tau_{c r}[\mathrm{MPa}]$} \\
\cline { 2 - 7 } & \multicolumn{4}{|c|}{ Numbers of terms in the series $m$} \\
\cline { 2 - 7 } & 3 & 5 & 7 & 9 & 11 & 21 \\
\hline \hline 100 & 480 & 479 & 479 & 479 & 479 & - \\
\hline 200 & 199 & 198 & 198 & 198 & 198 & - \\
\hline 500 & 171 & 143 & 142 & 142 & 142 & 140 \\
\hline 1000 & 199 & 145 & 132 & 131 & 131 & 131 \\
\hline 2000 & 285 & 199 & 159 & 138 & 133 & 129 \\
\hline
\end{tabular}

\section{References}

1. Adany S., 2018, Constrained shell Finite Element Method for thin-walled members, Part 1: constrains for a single band of finite elements, Thin-Walled Structures, 128, 43-55

2. Adany S., Visy D., Nagy R., 2018, Constrained shell Finite Element Method, Part 2: application to linear buckling of thin-walled members, Thin-Walled Structures, 128, 56-70

3. Hancock G.J., 2018, Coupled Instabilities in Metal Structures (CIMS) - What have we learned and where are we going? Thin-Walled Structures, 128, 2-11

4. Kolakowski Z., Krolak M., 2006, Modal coupled instabilities of thin-walled composite plate and shell structures, Composite Structures, 76, 303-313

5. Kolakowski Z., Mania J.R., 2013, Semi-analytical method versus the FEM for analysis of the local post-buckling, Composite Structures, 97, 99-106

6. Kolakowski Z., Teter A., 2000, Interactive buckling of thin-walled beam-columns with intermediate stiffeners or/and variable thickness, International Journal of Solids and Structures, 37, 3323-3344

7. Królak M., Kubiak T., KoŁakowski Z., 2001, Stability and load carrying capacity of thin-walled orthotropic poles of regular polygonal cross-section subject to combined load, Journal Theoretical and Applied Mechanics, 39, 4, 969-988 
8. Martins A., Landesman A., Camotim D., Dinis P., 2017, Distortional failure of cold-formed steel beams under uniform bending: Behaviour, strength and DSM design, Thin-Walled Structures, 118, 196-213

9. Martins A.D., Camotim D., Gonçalves R., Dinis P.B., 2018, GBT-based assessment of the mechanics of distortional-global interaction in thin-walled lipped channel beams, Thin-Walled Structures, 124, 32-47

10. Rendall M.A., Hancock G.J., Rasmussen K.J.R., 2017, The generalised constrained finite strip method for thin-walled members in shear, Thin-Walled Structures, 17, 294-302

11. Rendall M.A., Hancock G.J., Rasmussen K.J.R., 2018, Modal buckling behaviour of long polygonal tubes in uniform torsion using the generalised cFSM, Thin-Walled Structures, 128, $141-151$

12. Teter A., Kolakowski Z., 2003, Natural frequencies of thin-walled structures with central intermediate stiffeners or/and variable thickness, Thin-Walled Structures, 41, 4, 291-316

13. Teter A., Kolakowski Z., 2004, Interactive buckling and load carrying capacity of thin-walled beam-columns with intermediate stiffeners, The Special Issue of Thin-Walled Structures Journal "Cold-formed steel structures - Recent research advances in Central and East Europe", 42, 211-254 